\title{
Analyzing Network Coding (Gossip) Made Easy
}

\author{
BERNHARD HAEUPLER, Massachusetts Institute of Technology
}

\begin{abstract}
We introduce projection analysis—a new technique to analyze the stopping time of protocols that are based on random linear network coding (RLNC). Projection analysis drastically simplifies, extends, and strengthens previous results on RLNC gossip protocols. We analyze RLNC gossip in a general framework for network and communication models that encompasses and unifies the models used previously in this context. We show, in most settings for the first time, that the RLNC gossip converges with high probability in optimal time. Most stopping times are of the form $O(k+T)$, where $k$ is the number of messages to be distributed and $T$ is the time it takes to disseminate one message. This means RLNC gossip achieves "perfect pipelining."

Our analysis directly extends to highly dynamic networks in which the topology can change completely at any time. This remains true, even if the network dynamics are controlled by a fully adaptive adversary that knows the complete network state. Virtually nothing besides simple $O(k T)$ sequential flooding protocols was previously known for such a setting.

While RLNC gossip works in this wide variety of networks our analysis remains the same and extremely simple. This contrasts with more complex proofs that were put forward to give less strong results for various special cases.
\end{abstract}

Categories and Subject Descriptors: F.2.2 [Analysis of Algorithms and Problem Complexity]: Nonnumerical Algorithms and Problems-Computations on discrete structures; G.2.2 [Discrete Mathematics]: Graph Theory-Network problems; G.3.2 [Probability and Statistics]: Probabilistic Algorithms (including Monte Carlo)

General Terms: Algorithms, Performance, Reliability, Theory

Additional Key Words and Phrases: Random linear network coding, dynamic networks, gossip, multicast

ACM Reference Format:

Bernhard Haeupler. 2016. Analyzing network coding (gossip) made easy. J. ACM 63, 3, Article 26 (August 2016), 22 pages.

DOI: http://dx.doi.org/10.1145/2629696

\section{INTRODUCTION}

This article presents a new way to analyze protocols based on random linear network coding (RLNC), which we call projection analysis. Projection analysis substantially simplifies, extends, and strengthens the results of previous work on RLNC gossip protocols [Deb et al. 2005, 2006; Mosk-Aoyama and Shah 2006b; Vasudevan and Kudekar 2009; Borokhovich et al. 2010].

Gossip is a powerful tool to efficiently disseminate information. Its randomized nature is especially well suited to work in unstructured networks with unknown, unstable, or changing topologies. Because of this, gossip protocols have found a wide range

A preliminary version of this article appeared at the 2011 Symposium on Theory of Computing (STOC'11) [Haeupler 2011] where it was awarded the Danny Lewin Best Student Paper Award. This research was supported in part by NSF grant CCF-1618280 and NSF grant CCF-1527110.

Author's address: B. Haeupler, Computer Science Department, Carnegie Mellon University, Pittsburgh, PA 15213-3891; email: haeupler@cs.cmu.edu.

Permission to make digital or hard copies of part or all of this work for personal or classroom use is granted without fee provided that copies are not made or distributed for profit or commercial advantage and that copies show this notice on the first page or initial screen of a display along with the full citation. Copyrights for components of this work owned by others than ACM must be honored. Abstracting with credit is permitted. To copy otherwise, to republish, to post on servers, to redistribute to lists, or to use any component of this work in other works requires prior specific permission and/or a fee. Permissions may be requested from Publications Dept., ACM, Inc., 2 Penn Plaza, Suite 701, New York, NY 10121-0701 USA, fax +1 (212) 869-0481, or permissions@acm.org.

(c) 2016 ACM 0004-5411/2016/08-ART26 $\$ 15.00$

DOI: http://dx.doi.org/10.1145/2629696 
of applications [Haas et al. 2006; Mosk-Aoyama and Shah 2006a; Kempe et al. 2004; Demers et al. 1987; Agrawal et al. 1997] and have been extensively studied over the past several decades [Hedetniemi et al. 1988; Feige et al. 1990; Karp et al. 2000; Minski 2002; Kempe et al. 2003; Clementi et al. 2010; Aspnes and Ruppert 2007; Chierichetti et al. 2010; Giakkoupis 2011; Censor-Hillel et al. 2012a; Haeupler 2013; Giakkoupis et al. 2014; Haeupler and Malkhi 2014; Giakkoupis 2014].

Recently, gossip protocols based on random linear network coding [Ahlswede et al. 2000; Li et al. 2003; Ho et al. 2006] have been suggested [Deb and Médard 2004] to cope with the additional complexities that arise when multiple messages are to be distributed in parallel. RLNC gossip has been adopted in many practical implementations [Gkantsidis and Rodriguez 2005; Chou et al. 2003; Fragouli et al. 2008; Katti et al. 2008; Fragouli et al. 2006] and has performed extremely well in practice.

These successes stand in contrast to how little RLNC gossip is understood theoretically. Since its initial analysis on the complete graph [Deb and Médard 2004; Deb et al. 2005, 2006], several articles [Vasudevan and Kudekar 2009; Borokhovich et al. 2010; Mosk-Aoyama and Shah 2006b] have tried to give good upper bounds on the stopping time of RLNC gossip in more general topologies. However, none of them address the case of unstable or changing topologies, and, even with the restriction to static networks, the guarantees are far from being general or tight on most graphs. In addition, all existing proofs are quite involved and do not seem to generalize easily.

\section{Our Results}

This article has two main contributions. The first is our new projection analysis technique, which is both simpler and more powerful than previous approaches. Projection analysis relates the stopping time for $k$ messages to the much easier to analyze time $T$ needed to disseminate a single message. For the first time, and in practically all settings, this technique shows that RLNC gossip achieves perfect pipelining, that is, it disseminates $k$ messages in order optimal $O(T+k)$ time. Our results match, and in most cases improve, all previously known bounds and apply to more general models. To formalize this, we give a general framework for network and communication models that encompasses and unifies the models suggested in the literature so far. We give concrete results for several instantiations of this framework and give more detailed comparisons with previous results in each section separately.

As a second major contribution, our framework extends all models to (highly) dynamic networks in which the topology is allowed to completely change at any time. All of our results hold in these networks, even if the network dynamics are controlled by a fully adaptive adversary that decides the topology at each time, based on the complete network state as well as all previously used randomness. Virtually nothing, besides simple $O(k T)$ sequential flooding protocols [Kuhn et al. 2010], is known for such truly pessimistic network dynamics so far. Having optimal "perfectly pipelined" stopping times in worst-case adaptive dynamic networks is among the strongest stability guarantees for RLNC gossip that one might hope for. To this end, our results are the first that formally explain RLNC gossip performance in the dynamic environments it is used in and was designed for.

While the algorithm works in this wide variety of settings, our analysis remains mostly the same and extremely simple, in contrast with complex proofs that were previously put forward for the static setting.

\section{BACKGROUND AND RELATED WORK}

\subsection{Gossip}

Gossip is the process of spreading information via a randomized flooding procedure to all nodes in an unstructured network. It stands in contrast to structured multi-cast in 
which information is distributed via an explicitly built and maintained structure (e.g., a selection of spanning trees). While structured multi-cast can often guarantee optimal use of the limited communication resources, it relies heavily on having a known and stable network topology and fails in distributed or uncoordinated settings. Gossip protocols were designed to overcome this problem. By flooding information in a randomized fashion they guarantee to deliver messages with high probability to all nodes with little communication overhead. This stability and distributed nature of gossip makes it an important tool for collaborative content distribution, peer-to-peer networks, sensor networks, ad-hoc networks, and wireless networks; literature applying gossip in many areas and for many purposes is vast (e.g., Haas et al. [2006], Mosk-Aoyama and Shah [2006a], Kempe et al. [2004], Demers et al. [1987], and Agrawal et al. [1997]),

The gossip spreading of both a single message and multiple messages [Hedetniemi et al. 1988; Feige et al. 1990; Karp et al. 2000; Minski 2002; Kempe et al. 2003; Clementi et al. 2010; Aspnes and Ruppert 2007; Chierichetti et al. 2010; Giakkoupis 2011; Censor-Hillel et al. 2012a; Haeupler 2013; Giakkoupis et al. 2014; Haeupler and Malkhi 2014; Giakkoupis 2014] has been intensely studied. The spreading of one message often follows a comparatively simple epidemic random process in which the message is flooded to a randomly chosen subset of neighbors. Spreading multiple messages in parallel is significantly more complicated because nodes need to select which information to forward. The main problem in this context is that widely spread messages get forwarded more often and quickly outnumber rarer messages. In many cases the slow spread of the rare messages dominates the time needed until all nodes know every message.

\subsection{Network Coding}

A powerful and elegant way to avoid this and similar problems is the use of network coding techniques. Network coding as introduced by the seminal work of Ahlswede, $\mathrm{Cai}, \mathrm{Li}$, and Yeung [Ahlswede et al. 2000] breaks with the traditional concept that information is transported by the network as an unchanged entity. Ahlswede et al. show that in many multi-cast scenarios the optimal communication bandwidth can be achieved if and only if intermediate nodes in the network code information together. $\mathrm{Li}$ et al. [2003] showed that for multi-cast it is enough if intermediate nodes use linear coding, that is, computing linear combinations of messages. Following this, Ho et al. [2006] showed that the coefficients for these linear combinations need not be carefully chosen with regard to the network topology; indeed, using random linear combinations works with high probability for any fixed network.

The strong performance guarantees and the independence of the coding procedure from any global information about the network makes random linear network coding (RLNC) the perfect tool for spreading multiple messages. This was first observed and made formal by Deb and Médard [2004]. They show that using randomized gossip and RLNC in a complete network in which each of the nodes starts with one message, all information can be spread to all nodes in linear time, beating all non-coding approaches. After the introduction of this protocol in Deb and Médard [2004] and its follow-up [Deb et al. 2005, 2006], it was used in many applications [Chou et al. 2003; Fragouli et al. 2008; Katti et al. 2008; Fragouli et al. 2006], most notably the Microsoft Secure Content Distribution (MSCD) or Avalanche System [Gkantsidis and Rodriguez 2005]. There has also been more theoretical work [Vasudevan and Kudekar 2009; Borokhovich et al. 2010; Mosk-Aoyama and Shah 2006b] investigating the convergence time of RLNC gossip on general static network topologies. These are the most related works and we give a detailed description and comparison to these works in Section 6. 


\subsection{Dynamic Networks}

With mobile network devices becoming ubiquitous and many modern networks having an inherently large churn, robust communication protocols and the study of dynamic networks have gained importance. We refer to Kuhn et al. [2010] for an overview over the many different models that have been proposed to study these changing or instable topologies.

Even so, one one the main strengths of both gossip and network coding is their robustness, and this is the first study of network coding (gossip) in a dynamic setting. We build on and extend the dynamic network model from Kuhn et al. [2010] and Avin et al. [2008] in which an adaptive adversary chooses an arbitrary connected network topology at any time and in which nodes anonymously broadcast messages without knowing who their current neighbors are. Kuhn et al. [2010] presents multicast protocols based on flooding that demonstrate that even in this highly dynamic setting communication is possible. While these protocols occur a strong quadratic loss in performance compared to static networks the truly pessimal assumptions on the dynamics guarantee wide applicability.

In this work, we adapt this network model. We show that the robustness of network coding (gossip) is strong enough to cope even with this highly dynamic adversarial setting without any loss in efficiency. Building on this work, Haeupler and Karger [2011] showed that protocols using the network coding gossip approach presented here are able to perform provably better than any non-coding approach, that is, network coding beats lower bounds [Dutta et al. 2013; Haeupler and Kuhn 2012] established for protocols that merely try to route information.

\section{Organization}

Section 3 reviews the RLNC algorithm and Section 4 describes the new projection analysis technique. In Section 5 we introduce the network model framework. Section 6 shows how to apply the projection analysis in various instantiations of this framework. Section 7 discusses several ways in which the intentionally simple proofs from Section 6 can be extended or sharpened.

\section{THE RLNC ALGORITHM}

In this section, we give a brief description of the RLNC algorithm. The algorithm is simple and completely independent of the network structure or communication protocol. Alternative descriptions of the algorithm can be found in Deb and Médard [2004] or Chou et al. [2003].

The RLNC algorithm sends out packets in the form of vectors over a finite field $\mathbb{F}_{q}$, where $q$ is an arbitrary prime or prime power. We assume that there are $k$ messages, $\vec{m}_{1}, \ldots, \vec{m}_{k}$, that are vectors from $\mathbb{F}_{q}^{l}$ of length $l$. Every packet that is sent around during the execution of the algorithm has the form $(\vec{\mu}, \vec{m})$, where $\vec{m}=\sum_{i=1}^{k} \mu_{i} \vec{m}_{i} \in \mathbb{F}_{q}^{l}$ is a linear combination of the messages, and $\vec{\mu}=\left(\mu_{1}, \ldots, \mu_{k}\right) \in \mathbb{F}_{q}^{k}$ is the vector of the coefficients. If enough packets of this form are known to a node, that is, the span of the coefficient vectors is the full space $\mathbb{F}_{q}^{k}$, then Gaussian elimination can be used to reconstruct all messages. For this, only $k$ packets with linearly independent coefficient vectors are needed. Linearity furthermore guarantees that any new packet that is created by taking a linear combination of old packets has the same valid format. With this, it is easy to see that a node can produce any packet whose coefficient vector is spanned by the coefficient vectors of the packets it knows. The algorithm is now easily described.

Each node $v$ maintains a subspace $X_{v}$ that is the span of all packets known to it at the beginning and received so far by simply storing all received packets. If $v$ does not know any messages at the beginning, then $X_{v}$ is initialized to contain only the zero 
vector. If $v$ knows some message(s) $\vec{m}_{i}$ at the beginning, then $X_{v}$ is initialized to contain the packet $\left(\vec{\mu}, \vec{m}_{i}\right)$ in which $\vec{\mu}$ is the $i$ th standard basis vector. $X_{v}$ also contains all linear combinations that complete the span of these packet(s). Whenever node $v$ sends out a packet, it chooses a uniformly random packet from $X_{v}$ by taking a random linear combination of the stored packets. At the end of each round, all received packets are added to $X_{v}$, and again the span is taken. If the subspace spanned by the coefficient vectors is the full space, then a node decodes all messages.

Throughout the rest of the article we will solely concentrate on the "spreading" of the coefficient vectors; the linear combination of the messages implied by a coefficient vector $\vec{\mu}$ is always sent along with it. We therefore define $Y_{u}$ to be only the coefficient part of $X_{u}$, that is, the projection onto the first $k$ components.

Remark. The field size parameter $q$ is used to trade off a faster running time versus bandwidth overhead. While a larger $q$ can lead to faster convergence, it increases the communication overhead by increasing the size of the $k(\log q)$-size RLNC coefficients. In contrast to most related articles, all results in this article hold for arbitrary choices of $q$. For simplicity, we will often restrict ourselves to $q=2$. Note that this is the hardest case for runtime considerations and it can be safely assumed that convergence times for larger $q$ will only be better. The case $q=2$ is furthermore interesting because it leads to the minimal RLNC-coefficients overhead and allows the use of simple XORs as a basic arithmetic operation.

\section{THE PROJECTION ANALYSIS}

\subsection{Previous Approaches}

When analyzing the RLNC algorithm, Sub and Médard [Deb and Médard 2004] were the first to use the dimensionality of the subspaces $Y_{v}$ as a measure of progress. They defined a node $u$ to be helpful for a node $v$ if it knows something $v$ does not, that is, if the subspace $Y_{u}$ is not contained in $Y_{v}$. Whenever node $v$ receives a packet with a coefficient vector outside of $Y_{u}$ the dimension of $Y_{v}$ increases, which can happen at most $k$ times. More importantly, Sub et al. made the observation that if a node $u$ that is helpful to node $v$ sends this node a packet, then this dimension increase is very likely to happen. The reason for this is that the vectors $Y_{u} \cap Y_{v}$, which do not extend the dimensionality of $Y_{v}$, form a lower dimensional subspace in $Y_{u}$. Thus, whenever a node $u$ sends a random vector from $Y_{u}$ to a node $v$ it is helpful for, the probability that the dimension of $Y_{v}$ increases is at least $1-1 / q$. This fact and the notion of helpfulness is used as a crucial tool in all further RLNC proofs [Deb et al. 2005, 2006; Vasudevan and Kudekar 2009; Borokhovich et al. 2010; Mosk-Aoyama and Shah 2006b].

\subsection{Our Analysis Technique}

We argue that the right way to look at the spreading of information is to look at the orthogonal (dual) complement $Y_{u}^{\perp}$ of the coefficient subspaces $Y_{u}$. While the coefficient subspaces grow monotonically to the full space, their orthogonal complement decreases monotonically to the empty span. To see how quickly this happens, we first concentrate on one fixed (dual) vector $\vec{\mu} \in F_{q}^{k}$, determine the time that is needed until it disappears from all subspaces $Y_{u}^{\perp}$ with high probability, and then take a union bound over all those dual vectors.

A different and maybe even simpler way of looking at this is that we are analyzing the spreading process by looking at its $q^{k}$ one-dimensional projections. To keep track of these projections, we introduce the following crucial notion of knowing:

Definition 4.1. A node $u$ knows about $\vec{\mu} \in \mathbb{F}_{q}$ if its coefficient subspace $Y_{u}$ is not orthogonal to $\vec{\mu}$, that is, if there is a vector $\vec{c} \in Y_{u}$ with $\langle\vec{c}, \vec{\mu}\rangle \neq 0$. 
The intuition behind this notion is that a node that has not received any message in which the direction $\vec{\mu}$ was "mixed in" is completely ignorant about this direction while any other node "knows" at least something about $\mu$. In particular, it knows at least one of the many packets that have a non-orthorgonal coefficient vector to $\vec{\mu}$. We remark that this "not being completely ignorant" condition is very weak. In particular, a node $u$ knowing a vector $\vec{\mu}$ does not imply $^{1} \vec{\mu} \in Y_{u}$ or anything about $u$ being able to decode a message associated with the coefficients $\vec{\mu}$. On the other hand, this weak condition makes it easy to prove that knowledge spreads very easily. It is also strong enough that knowing all (exponentially many) vectors in $\mathbb{F}_{q}^{k}$ is sufficient for decoding. These two useful facts are shown in the next lemma.

LEMMA 4.2. If a node $u$ knows about a vector $\vec{\mu}$ and transmits a packet to node $v$, then $v$ knows about $\vec{\mu}$ afterwards with probability at least $1-1 / q$. Furthermore, if a node knows about all vectors in $\mathbb{F}_{q}^{k}$, then it is able to decode all $k$ messages.

Proof. Knowledge about a $\vec{\mu}$ spreads with a transmission with probability $1-$ $1 / q$ because the vectors in $Y_{u}$ that are perpendicular to $\vec{\mu}$ form a lower-dimensional hyperplane with at most a $1 / q$ fraction of the volume.

For the second claim, we prove the counterpositive, namely that any node $u$ that is not able to decode does not know at least one vector $\vec{\mu}$ : If $u$ cannot decode, then $Y_{u}$ is not the full space. Because $Y_{u}$ is a subspace, it is lower dimensional and there is a vector $\vec{\mu}$ that is orthogonal to $Y_{u}$. This vector $\vec{\mu}$ is then, by definition, not known to $u$.

Lemma 4.2 implies that the spreading of knowledge for a fixed vector $\vec{\mu} \in F_{q}^{k}$ is a monotone increasing, epidemic set growing process or, differently phrased, each projection looks essentially like a $1 / q$-faulty one-message flooding process. It is usually relatively easy to understand this process and to determine the expected time $T$ until all nodes know $\vec{\mu}$. Furthermore, because the set growing process is a monotone Markov chain, its stopping probability has an exponentially decaying tail. In most cases, this tail kicks in close to the expectation. This allows us to pick a time $t$ (usually $t=O(T+k)$ ) after which any vector in $\mathbb{F}_{q}^{k}$ has spread with probability at least $1-q^{-\Theta(k)}$. Taking a union bound over all $q^{k}$ vectors then completes the proof that with high probability everything has spread. The following definition and theorem summarizes these ideas.

Definition 4.3 (Faulty One-Message Broadcast). For any network and communication model whose dynamics do not depend on what nodes know and for any $p \geq 0$, its $p$-faulty one-message broadcast is defined as follows: One message starts at some node $v$, and in every round, every node that knows the message and is supposed to communicate according to the communication model forwards it with probability $1-p$ and remains silent otherwise.

THEOREM 4.4. Fix a prime (power) $q \geq 2$, a probability $\delta>0$, and an arbitrary network and communication model whose dynamics do not depend on what nodes know. If, for its $1 / q$-faulty one-message broadcast starting from any node $v$, the probability that the message reaches all nodes after $t$ rounds is at least $1-\delta q^{-k}$, then $k$ messages can be spread in the same model in time t with probability $1-\delta$ using the RLNC gossip protocol with field size $q$.

\footnotetext{
${ }^{1}$ Counterintuitively, because we are not working over a positive-definite inner-product space, it can even be that $\vec{\mu} \in Y_{u}$ but $u$ does not know $\vec{\mu}$ according to the above definition. For example, in $F_{2}^{2}$, the vector $(1,1)$ is orthogonal to itself and a node having only received a packet with the $(1,1)$ coefficients would not know $\mu=(1,1)$.
} 
Proof. The theorem follows directly from the discussion above and Lemma 4.2. Initially every non-zero vector $\vec{\mu} \in \mathbb{F}_{q}^{k}$ is known to at least one node, namely the one that knows about the $i$ th message, where $i$ is a non-zero component of $\vec{\mu}$. Whenever the network and communication model dictates that a node $u$ that knows $\vec{\mu}$ sends a message to a node $v$, Lemma 4.2 shows that with probability $1-1 / q$ the node $v$ afterwards knows $\vec{\mu}$. Therefore, the spreading of each vector $\vec{\mu}$ behaves like a faulty flooding process that floods $\vec{\mu}$ in every transmission with probability $1-1 / q$. By assumption, we have that after $t$ time steps every vector from $\mathbb{F}_{q}^{k}$ fails to spread to all nodes with probability at most $\delta q^{-k}$. Taking a union bound over all $q^{k}$ vectors gives the guarantee that the probability that after $t$ rounds all nodes know all vectors is at least $1-\delta$. According to Lemma 4.2, all nodes can decode in this case and have learned the $k$ messages.

\subsection{A Typical Template}

Next, we give a typical and easy way to apply Theorem 4.4. We show that, even for $q=2$, the time for one vector $\vec{\mu}$ to spread is often dominated by a negative binomial distribution $N B(T, 1-p)$, where $T$ is the expected time to spread one message and $p$ is a constant probability. Such a distribution has a strong-enough tail to prove optimal $O(T+k)$ stopping times. In what follows, we give a simple template for this argument.

What is needed for this template is a definition of a "successful round," such that at most $T$ such rounds are needed to spread a single vector $\vec{\mu}$ and such that a round is not a success with probability at most $p$. The appropriate definition of success depends on the network model and is usually centered around how many additional nodes come to know the vector in a "good round."

Since nodes do not forget any information, this spreading process is monotone, that is, no progress gets lost in a bad round. Thus, if the knowledge about $\vec{\mu}$ has not spread after $t=c\left(k+T+\log \delta^{-1}\right)$ steps, then there were at least $c\left(k+T+\log \delta^{-1}\right)-T>$ $(c-1)\left(k+T+\log \delta^{-1}\right)$ failures, whereas one would only expect $p c\left(k+T+\log \delta^{-1}\right)$. If we choose the constant $c$ large enough, then a standard Chernoff bound implies that the probability for this to happen is at most $2^{-\Theta\left(k+T+\log \delta^{-1}\right)}$. This is small enough that, after a union bound over all $q^{k}$ vectors (e.g., for $q=2$ ), the probability that all $k$ messages have not spread is at most $\delta$.

This is usually all that is needed to prove order optimal $O(k+T)$ stopping times. It also shows that we actually obtain high-probability results. In particular, as shown here, an additive $\Theta\left(\log \delta^{-1}\right)$ additional round typically suffices to obtain a $1-\delta$ success probability for any $\delta>0$. This strong and optimal guarantee is (up to some scaling) true for all our results; even so, we do not state it explicitly for sake of simplicity.

\section{NETWORK MODEL AND COMMUNICATION FRAMEWORK}

In this section, we elaborate on our network model framework that encompasses and extends the models suggested in the literature so far. The models and the results can be easily extended further; we chose the following framework as a tradeoff between simplicity and generality.

\section{The Network}

We consider networks that consist of $n$ nodes. A network is specified by a (directed) graph $G(t)$ on these nodes for every time $t$. Edges in $G(t)$ are links and present potential communication connections between two nodes in round $t$. We will usually assume that the network has, at all times, certain connectivity properties and will express the stopping time in terms of these parameters. (See also Section 7.4.) 


\section{(Adversarial) Dynamics:}

In all previous articles that analyzed the RLNC algorithm, the network topology was assumed to be static, that is, $\forall t: G(t)=G$. As discussed in the Introduction, we allow the network topology to change completely from round to round and allow a fully adaptive adversary to choose the network. Because we are dealing with randomized protocols, we have to specify precisely to what the adversary is allowed to adapt. In our models (similar to Kuhn et al. [2010]), an adaptive adversary gets to know the complete network state and all previously used randomness when choosing the topology. After that, independent randomness is used to determine the communication behavior and the messages of the nodes on this topology. The last assumption is not essential; in further work [Haeupler and Karger 2011], it was shown that, for a large-enough $q$, the analysis presented here works even against omniscient adversaries that get to know all randomness used for the coding coefficients in advance.

\section{The Goal: Gossip}

Distributed over the network are $k$ messages numbered $1, \ldots, k$, each known to at least one node. Throughout this article, we assume a worst-case starting configuration for all messages including the case in which all messages are exclusively known to only one node (see also Section 7.1). The goal of gossip protocols is to make all messages known to all nodes in the network using as little time as possible (in expectation and with high probability)

\section{Communication}

Nodes communicate along links during transactions that are atomic in time. In each round, one packet is transmitted over a link if this link is activated in this round. From the view of a node there are four commonly considered types of connections. Either a node sends to all its neighbors, which is usually referred to as BROADCAST, or it establishes a connection to one (e.g., uniformly random) neighbor and sends (PUSH) or receives (PULL) a message or both (EXCHANGE). In all cases, the packet is chosen without the sender knowing which node(s) will receive it.

\section{Message and Packet Size}

As described in Section 3, we assume that all messages and packets have the same size and that a packet exactly contains one encoded message and its RLNC-coefficients. Note that the restriction on the message size is without loss of generality, since one can always cut a big message into multiple messages that fit into a packet. We also assume that the message size is large enough that the size of the RLNC coefficients that are sent along is negligible, that is, $l \gg k$. This assumption was made by all previous work and is justified by simulations and implementations in which the overhead is only a small fraction (e.g., < 1\% [Deb and Médard 2004]) of the packet size.

\section{Synchronous versus Asynchronous Communication}

We consider two types of timing models. In the synchronous case, all nodes get activated at the same time and choose their messages independently, and messages get delivered according to the current network $G(t)$ and who sends and receives from whom. Note that this model is inherently discrete, and we assume that $t=1,2, \ldots$, are the times when nodes communicate. For the asynchronous case, we assume that every node communication is triggered independently by a Poisson clock. This means that (with probability one) at any time only one node sends its message. This model can be directly translated into a discrete time model that defines round $i$ as the $i$ th time such a communication takes place. The model considered in the literature so far assumes that every node is activated uniformly at random to communicate and then chooses a uniformly random neighbor for a PUSH, PULL, or EXCHANGE. They also scale the 
time in the asynchronous model by a factor of $1 / n$ so each node gets activated once per time unit in expectation. We do not assume uniformity in either of the two distributions, and we present results for this more general model in Section 6.2.

\section{APPLICATIONS AND RESULTS}

In this section we take the models from Section 5 and describe the results that can be obtained for them using the projection analysis technique. There is a section for each communication model. We start with the Random Phone Call Model [Deb and Médard 2004 ] that introduced RLNC gossip. We than cover the extensions to arbitrary underlying network topologies as considered by Vasudevan and Kudekar [2009], Borokhovich et al. [2010], and Mosk-Aoyama and Shah [2006b]. Section 6.2 proves stopping times for a communication model that encompasses all former asynchronous communication protocols (PUSH, PULL, EXCHANGE, ...). For this model, we answer a question of Borokhovich et al. [2010] and show that a simple min-cut quantity exactly captures the behavior of gossip of $n$ messages. Last, in Section 6.3, we give the first bounds for the performance of synchronous and asynchronous BROADCAST. We concentrate on showing simple proofs that solely use the template from Section 4.3. In Section 7, we revisit the models covered here and discuss some proof extensions.

\subsection{Random Phone Call Model and Rumor Mongering}

In this section, we consider the work of Deb and Médard [2004] and its follow-up [Deb et al. 2005, 2006] and show how to simplify and improve the analysis. The articles use a fairly simple model from our framework, namely the synchronous PUSH or PULL model on the complete graph, that is, $G(t)=K_{n}$. This means in each round each node picks a random other node to exchange information with. This model is also known as rumor mongering or the random phone call model and was introduced by Demers et al. [1987]. It is shown in Deb and Médard [2004] that it is possible in this model to spread $k=\Theta(n)$ messages in $O(n)$ time if $q=n$. This beats the $O(n \log n)$ time of $n$ sequential $O(\log n)$ phases of flooding just one message. The follow-up articles [Deb et al. 2005, 2006] generalize this result to smaller number of messages $k$ and allow $q$ to be as small as $k$. They show that the running time of the algorithm is $t=O(k+\sqrt{k} \log k \log n)$, that is, order optimal as long as $k \geq \log ^{2+\epsilon} n$ for any $\epsilon>0$. In order to prove this result, they have to assume that each node knows initially only one message and that initially the messages are equally spread. Even with these assumptions the analysis is long and complicated and the authors state themselves in their abstract that "While the asymptotic results might sound believable, owing to the distributed nature of the system, a rigorous derivation poses quite a few technical challenges and requires careful modeling and analysis of an appropriate time-varying Bernoulli process."

Our next lemma shows that RLNC gossip actually finishes with high probability in order optimal stopping time $O(k+\log n)$. Our analysis is much simpler and has many further advantages: It holds for all choices of $k$ and allows $q$ to be as small as 2 . The proof is (almost) the same for all communication models while two completely separate proofs for the PUSH and the PULL protocol had to be given in previous works. Furthermore, our proof does not rely on any assumptions on the initial message distribution. This is important since we show in Section 7.2 that the well-mixed initial state assumed in Deb and Médard [2004] and Deb et al. [2005, 2006] actually provably speeds up the stopping time compared to the worst-cast distribution for which our result holds. Our proof gives a success probability of $1-2^{t}$ if the algorithm runs for $O(t)$ time. In the setting of Deb and Médard [2004] with $k=n$, this is $1-2^{-n}$ instead of the $1-1 / n$ stated there. Last, it is interesting to note that later, more general approaches like those of Borokhovich et al. [2010] and Mosk-Aoyama and Shah [2006b] were unable to prove any running time that beats the simple non-coding $O(n \log n)$ sequential flooding 
approach when applied to the complete graph ([Borokhovich et al. 2010] even gives only a $O\left(n^{2}\right)$ convergence time).

LEMMA 6.1. The RLNC gossip in the random phone call model with $q=2$ spreads $k$ messages with high probability in exactly $\Theta(k+\log n)$ time. This holds independently from the initial distribution of the messages and of the communication model (e.g., PUSH, PULL, EXCHANGE).

Proof. We use the template from Section 4.3: For this we fix a coefficient vector $\vec{\mu}$ and define a round as successful if the number of nodes that know it increases by at least a constant factor $\lambda>1$ or if the number of nodes that do not know $\vec{\mu}$ decreases by a factor of $\lambda$. There are at most $O(\log n)$ successful rounds needed until at least $n / 2$ nodes know $\vec{\mu}$ and at most another $O(\log n)$ successful rounds until all nodes know $\vec{\mu}$. It remains to be shown that each round succeeds with constant probability.

We start with the PULL model. At first, we have $i<n / 2$ nodes that know $\vec{\mu}$ and at least $n / 2$ nodes pulling for it. Each of those nodes has a probability of $i / n$ to hit a knowing node. We expect a $i / n$ fraction of the ignorant nodes, that is, at least $i / 2$ nodes, to receive a packet from a node that knows about $\vec{\mu}$. The independence of these successes and Lemma 4.2 prove that with constant probability at least $\Omega(i)$ nodes learn about $\vec{\mu}$. Once there are at least $n / 2$ nodes that know $\vec{\mu}$, each of the ignorant nodes pulls a packet from a knowing node with probability at least $1 / 2$ and applying Lemma 4.2 again finishes this case, too.

The proof for the PUSH model is similar. If there are $i<n / 2$ nodes that know $\vec{\mu}$ and push out a packet, then there are at least $n / 2$ ignorant nodes that each receive at least one packet from one of the $i$ nodes with probability $1-(1-1 / n)^{i}$. It is not hard to see that, in total, $\Omega(i)$ ignorant nodes receive a packet from a node that knows $\vec{\mu}$ with constant probability. Lemma 4.2 now guarantees that, with constant probability, the number of ignorant nodes that learn $\vec{\mu}$ is only a small factor smaller. Once there are $n / 2$ nodes knowing $\vec{\mu}$ and each of these pushes out, each node that does not know $\vec{\mu}$ has a chance of $(1-1 / n)^{n / 2} \approx e^{-2}$ per round to receive a packet from a node that knows $\vec{\mu}$. Applying Lemma 4.2 again finishes the proof of the upper bound.

For the lower bound we note that each node receives in expectation (and in the long run with high probability) only $\Theta(1)$ packets per round. Thus, if in the beginning at least one node did not already know a constant fraction of the messages, then the algorithm has to run for at least $\Omega(k)$ rounds. It is also clear that even just one message starting in one node takes in expectation $\Omega(\log n)$ time to spread to all nodes.

\subsection{Asynchronous Single Transfer Protocols}

After the helpfulness of RLNC gossip was established for the complete graph by Deb and Médard [2004], the articles by Mosk-Aoyama and Shah [2006b], Vasudevan and Kudekar [2009], and [Borokhovich et al. 2010] generalized it to general static topologies and consider asynchronous and synchronous PUSH, PULL, and EXCHANGE gossip. In this section, we first review the previous results and then show how to improve them giving an exact characterization of the stopping time or RLNC gossip for $k=n$ messages using the template of Section 4.3.

The article "Information Dissemination via Network Coding" by Mosk-Aoyama and Shah [2006b] was the first to consider general topologies. They consider a similarly general version of the synchronous and asynchronous gossip as presented here and analyze the stopping times for $k=n$ in dependence on the conductance. Their analysis implies that, with high probability, $O(n \log n)$ phases of $n$ asynchronous rounds suffice for the complete graph and constant degree expanders and that $O\left(n^{2}\right)$ such phases are sufficient for the ring graph. While the analysis is very interesting, these results do not beat the simple (non-coding) sequential flooding protocol, and the stopping time of 
the ring graph and many other graphs is even off by a factor of $n$. Their running times for the synchronous model are similar but loose another $\log n$-factor. Their dependence is on the success probability $1-\delta$ is furthermore multiplicative in $\log \delta^{-1}$ because it stems from a standard probability amplification argument.

More recently, Vasudevan and Kudekar [2009] and Borokhovich et al. [2010] also analyzed RLNC gossip using two completely different approaches. The authors of Borokhovich et al. [2010] point out that the analysis of Vasudevan and Kudekar [2009] is flawed and prove that the asynchronous RLNC gossip on a network with maximum degree $\Delta$ takes with high probability $O\left(\Delta n^{2}\right)$ time (using the scaling of time used in this article). Their proof uses an interesting reduction to networks of queues and applies Jackson's theorem. They also give a tight analysis and lower bounds for a few special graphs with interesting behavior (see below). While their analysis is exact for few selected graphs the analysis is far from tight; in most graphs the maximum degree has nothing to do with the stopping time of RLNC gossip. The major question asked in Borokhovich et al. [2010] is to find a characterizing property of the graph that determines the stopping time.

We give such a characterization for the asynchronous case with $k=n$ assuming a worst-cast message initialization. The model we use is a generalization of the classical PUSH, PULL, and EXCHANGE model: We allow the topology in every round to be specified by a graph with directed and/or undirected edges and a probability weight $p_{e}$ on every edge $e$, such that the sum over all edges is at most 1 . In every round each edge gets exclusively selected with probability $p_{e}$, that is, in each round at most one edge gets selected. If the edge is undirected, then an EXCHANGE is performed and if a directed edge gets activated, then a packet is delivered in the direction of the edge. Note that this model is a generalization of the "classical" communication models. To obtain the probability graph from an undirected network with PUSH or PULL, one just has to replace every undirected edge $\{u, v\}$ by two directed edges with probability weight $\frac{1}{n \Delta_{u}}$ and $\frac{1}{n \Delta_{v}}$, where $\Delta_{u}$ and $\Delta_{v}$ are the degrees of $u$ and $v$, respectively. To obtain the EXCHANGE protocol each undirected edge $\{u, v\}$ simply has the probability weight $\frac{1}{n \Delta_{u}}+\frac{1}{n \Delta_{v}}$.

Given such a network graph $G$ with probability weights $p_{e}$ we define the min-cut $\gamma(G)$ as:

$$
\gamma(G)=\min _{\emptyset \neq S \subset V} \sum_{e \in \Gamma_{G}^{+}(S)} p_{e},
$$

where $\Gamma_{G}^{+}(S)$ are all edges leaving a non-empty vertex-subset $S \subset V$ in $G$. The next two lemmas show that this quantity exactly captures how long the RLNC gossip takes to spread $n$ messages.

LEMMA 6.2. If for every time the min-cut of $G(t)$ is at least $\gamma$ then the asynchronous single transfer algorithm with $q=2$ spreads $n$ messages with probability at least $1-2^{-n}$ in $O\left(\frac{n}{\gamma}\right)$ time.

PRoof. Our proof proceeds along the lines of the simple template from Section 4.3 and concentrates on the spreading of one coefficient vector. We define a round as a success if and only if one more node learns about it. It is clear that exactly $n$ successes are needed. From the definition of $\gamma$ and Lemma 4.2, it follows that each round is successful with probability of at least $\gamma(1-1 / q)$. Thus, if we run the protocol for $t=c\left(\frac{n}{(1-1 / q) \gamma}\right)$ rounds, then we expect at least $c n$ successes and by Chernoff bound the probability that we get less than $n$ is at most $2^{-\Theta(n)}$. If we choose $c$ appropriately, then this is small enough to end up with $2^{-n}$ after taking the union bound over the $q^{k}=2^{n}$ vectors. 
The next lemma proves that $O\left(\frac{n}{\gamma}\right)$ is optimal.

LEMMA 6.3. With high probability, the asynchronous single transfer algorithm takes at least $\Omega\left(\frac{k}{\gamma}\right)$ rounds to spread $k$ messages if it is used on any fixed graph $G$ with (min-)cut $\gamma$ on which at least $\Theta(k)$ messages are initialized inside this cut.

Proof of Lemma 6.3. In each round, at most one packet can cross the cut. For this to happen, an edge going out of the cut has to be selected and the probability for this is by definition exactly $\gamma$. In order to be able to decode the $k$ messages, at least $\Theta(k)$ packets have to cross the cut each taking in expectation at least $O\left(\frac{1}{\gamma}\right)$ rounds. A standard Chernoff bound shows that it takes with high probability at least $\Omega\left(\frac{k}{\gamma}\right)$ rounds until $\Theta(k)$ packets have crossed the cut.

Applying Lemma 6.2 to the standard PUSH/PULL model gives a $O\left(\Delta n^{2}\right)$ stopping time for any dynamic graph whose maximum degree is bounded by $\Delta$, which is the main result of Borokhovich et al. [2010] (see also [Haeupler 2012]). It also gives $O\left(n^{2}\right)$ for the complete graph (instead of the $O\left(n^{3}\right)$ of Borokhovich et al. [2010]) and nicely explains the behavior of the barbel graph and the extended barbel graph that were considered by Borokhovich et al. [2010]. The proof of Lemma 6.2 can furthermore easily be extended to show that the dependency on the success probability is only logarithmic and additive in contrast to the previous work [Mosk-Aoyama and Shah 2006b; Borokhovich et al. 2010].

\subsection{Broadcast}

In this section, we give stopping times for synchronous and asynchronous BROADCAST protocols in arbitrary dynamic networks. These are, to our knowledge, the first results for the RLNC algorithm in such a setting. We think the results in this section are of particular interest for highly dynamic networks because many of the unstable or dynamic networks that occur in practice, like ad-hoc, vehicular, or sensor networks, are wireless and thus have an inherent broadcasting behavior.

To fix a model, we first consider the simple synchronous broadcast model. We assume without loss of generality that the network graph $G$ is directed because any undirected edge can be replaced by two directed, anti-parallel edges. Having wireless networks in mind, we also assume that in each round each nodes computes only one packet which is then send out to all neighbors. Our results also hold for the easier but usually less realistic model where nodes send out a different packet to each neighbor.

The parameter that governs the time to spread one message in a static setting is (not surprisingly) the diameter $D$, and it is easy to prove $\Theta(D+k)$ stopping times for $k$ messages using the projection analysis. In a dynamic setting, this is not true. Even for just one message, an adaptive adversary can, for example, always connect both the set of nodes that know it and the set of nodes that do not know it to a clique, and connect the two cliques by one edge. Even though the graph $G(t)$ has diameter 3 at all times, it clearly takes at least $n$ rounds to spread one message. In order to prove stopping times in the adaptive adversaries model, we switch to a parameter that indirectly gives a good upper bound on the diameter for many graphs. The parameter we use is the isoperimetric number $h(G)$, which measures the "node expansion" of a graph as follows:

$$
h(G):=\min _{\emptyset \neq S \subset V} \frac{\left|N_{G}^{+}(S)\right|}{\min (|\bar{S}|,|S|)},
$$

where $N_{G}^{+}(S)$ are the nodes in $G$ outside of the subset $S$ that are in the directed neighborhood of $S$. 
To give a few example values: For disconnected graphs $h(G)$ is zero, and for connected graphs it ranges between 1 and $\frac{2}{n}$; for a $k$-vertex-connected graph $G$ we have $h(G)=$ $\Omega\left(\frac{k}{n}\right)$, and $h(G)=\Theta(1)$ holds if and only if $G$ is a vertex expander (or a complete graph).

We are going to show that the expected time for one message to be broadcast is at most $T=\frac{\log (n h(G))}{h(G)}$. This is $O(n)$ for a line or cycle, $O\left(\frac{n \log k}{k}\right)$ for a $k$-vertex-connected graph, and $O(\log n)$ for any vertex expander. Our bound is tight in the sense that for any value $h$ with $1 \geq h \geq \frac{2}{n}$ there is a static graph $G$ that has diameter at least $O(T)$ and isoperimetric number $h(G)=\Theta(h)$. Having an upper bound on the time $T$ it takes to spread one message, we again prove a perfectly pipelined time of $O(T+k)$ for $k$ messages:

LEMMA 6.4. The synchronous broadcast gossip protocol takes with high probability at most $O\left(\frac{\log (n h)}{h}+k\right)$ rounds to spread $k$ messages as long as the isoperimetric number of the graph $G(t)$ is at least h at every time $t$.

Proof. We use the simple template from Section 4.3 and concentrate on the spreading of one coefficient vector $\vec{\mu}$. We define a round to be a success if and only if the number of nodes that know $\vec{\mu}$ grows at least by a $\frac{h}{7}$ fraction or if the number of nodes that do not know $\vec{\mu}$ shrinks at least by the same factor.

We want to argue that at most $T=O\left(\frac{\log (n h)}{h}\right)$ successes are needed to spread $\vec{\mu}$ completely. Note that this is slightly better than the straightforward $\left(1+\frac{h}{7}\right)^{T} \geq n$ bound that would lead to $T=O\left(\frac{\log (n)}{h}\right)$. The improvement comes from exploiting the fact that the number of nodes that learn is an integral quantity: In the first $\frac{7}{h}$ successful rounds, at least one node learns about $\vec{\mu}$. In the next $\frac{7}{2 h}$ successful rounds, at least 2 nodes learn about $\vec{\mu}$, and in the following $\frac{7}{3 h}$ successful rounds it is 3 new nodes and so on. There are $\frac{n}{2} \cdot\left(\frac{7}{h}\right)^{-1}$ such phases until at least $n / 2$ nodes know $\vec{\mu}$. The downward progression then follows by symmetry. The total number of successes sums up to:

$$
T \leq 2 \frac{7}{h} \sum_{i=1}^{O(n h)} \frac{1}{i}=O\left(\frac{\log n h}{h}\right) .
$$

To finish the proof, we show that every round has a constant success probability. This follows from Lemma 4.2 if for a success only one node is supposed to learn about $\vec{\mu}$. If at least $\lceil i\rceil \geq 2$ nodes are supposed to learn, then by the definition of a success and of $h(G(t))$ there are $k \geq\lceil 7 i\rceil \geq 4\lceil i\rceil$ nodes on the knowledge cut, that is, at least $k$ nodes that do not know $\vec{\mu}$ are connected to a node that knows about $\vec{\mu}$. We invoke Lemma 4.2 again to see that each of these nodes fails to learn about $\vec{\mu}$ with probability at most $1 / q \leq 1 / 2$. Finally, Markov's inequality gives that the probability that more than $k-\lceil i\rceil \geq \frac{3}{4} k$ nodes fail to learn $\vec{\mu}$ is at most $2 / 3$. A round is therefore successful with a probability of at least $1 / 3$.

A similar result to Lemma 6.4 can be proven for the asynchronous BROADCAST model in which at every round each node gets selected uniformly independently at random (i.e., with probability $\frac{1}{n}$ ) to broadcast its packet to its neighbors.

LEMMA 6.5. The asynchronous broadcast gossip protocol takes with high probability at most $O\left(n \cdot\left(\frac{\log (n h)}{h}+k\right)\right)$ rounds to spread $k$ messages as long as the isoperimetric number of the graph $G(t)$ is at least $h$ at any time $t$.

Proof. The proof is nearly identical to the one of Lemma 6.4, but instead of defining a round as a success we define successes for phases of $n$ consecutive rounds. Using the 
same definition of success and following the same reasoning as before, it is clear that at most $O\left(\frac{\log (n h)}{h}\right)$ successful phases are needed. To finish the proof, we have to show that every phase has a constant success probability. For this we note again that at least $k \geq 4\lceil i\rceil$ nodes are on the knowledge-cut of $\vec{\mu}$ if $\lceil i\rceil$ nodes need to learn about $\vec{\mu}$. For each of these $4\lceil i\rceil$ nodes the probability that no neighboring node that knows $\vec{\mu}$ is activated during $n$ rounds is at most $(1-1 / n)^{n}=e^{-1}$. According to Lemma 4.2 the probability for each of the $k$ nodes to fail to learn about $\vec{\mu}$ is thus at most $1-(1-1 / q)\left(1-e^{-1}\right)<$ $0.7<3 / 4$. Markov's inequality again implies that the probability for a failed round in which more than $k-\lceil i\rceil \geq 3 / 4 k$ fail is at most $0.7 / 0.75$.

\section{EXTENSIONS}

In this section, we discuss how the simple proofs from Section 6 that use only the template from Section 4.3 can be extended to give more detailed or sharper bounds.

\subsection{Exploiting a Well-Mixed Message Initialization}

As stated in Section 5, we assume throughout the article that $k$ messages are to be spread that are initially distributed in a worst-case fashion. All earlier articles restricted themselves to the easier special case that $k=n$ and that each node initially holds exactly one message [Borokhovich et al. 2010; Mosk-Aoyama and Shah 2006b] or that $k$ is arbitrary but the network starts in a similarly well-mixed state in which each message is known by a different node and all messages are equally spread over the network [Deb et al. 2006]. In many cases the worst-case and any well-mixed initialization take equally long to converge because the running time is lower bounded and bottlenecked by the flooding time $T$ for a single message or the time it takes for a node to receive at least $k$ packets. Nevertheless, there are cases where a well-mixed initialization can drastically improve performance.

Our proof technique explains this and we give a simple way to exploit assumptions about well-mixed initializations to prove stronger performance guarantees: If, for example, each node initially holds exactly one of $k=n$ messages, then most vectors $\vec{\mu}$ are already known to most nodes initially. More precisely, exactly the $\left(\begin{array}{c}n \\ i\end{array}\right)(q-1)^{i}$ vectors with $i$ non-zero components are initially known to exactly $i$ nodes. With many vectors already widely spread initially, the union bound over the failure probabilities for all vectors to spread after $t$ rounds can decrease significantly. Taking the different quantities and probabilities for nodes that are initially known to a certain number of nodes in account one can prove in theses cases that a smaller $t$ suffices.

One example for a mixed initialization being advantageous is discussed in Section 7.2. Another example are the convergence times of the asynchronous PUSH and PULL protocol on the star graph: For both PUSH and PULL, the network induced by the star graph has a min-cut of $1 / n^{2}$, which leads according to Lemma 6.2 and 6.3 to a stopping time of $\Theta\left(n^{3}\right)$ under a worst-case initialization. The corresponding lower bound from Lemma 6.3, which relates the convergence time to the min-cut of the network graph, has to assume that at least a constant fraction of the messages are initialized inside a bad cut. For the "classical" initialization in which each node starts with exactly one message this is true for the PUSH model but not in the PULL model in which every bad cut only contains few messages. Indeed, assuming a well-mixed initialization, the PUSH protocol still takes $\Theta\left(n^{3}\right)$ time to converge while a much lower $\Theta\left(n^{2} \log n\right)$ stopping time for the PULL model can be easily derived using the projection analysis.

\subsection{Perfect Pipelining and Exact Dependence on $\boldsymbol{k}$}

In many (highly connected) networks the spreading time $T$ for one message is short and $O(k)$ becomes the dominant term in the order optimal $O(k+T)$-type upper bounds 
presented in this article. So is, for example, $T=O(\log n)$ for most expanding networks. While it is clear that at least $k$ packets need to be received at each node it becomes an interesting question how large the constant factor hidden by the $O$-notation is. Differently stated, we ask how large the fraction of helpful or innovative packets received by a node is over the execution of the protocol.

Determining and even more optimizing proofs to obtain such constants is usually a big hassle or even infeasible due to involved proofs. Simulation is therefore often used in practice to get a good estimation of the constants (e.g., Deb et al. [2006]). Our template from Section 4.3 reduces the question for the stopping time of RLNC gossip to a simple standard question about tail bounds for negative binomial random variables. This makes it often possible to determine and prove (optimal) constants (and lower order terms). All that is needed is to replace the Chernoff bound in the template from Section 4.3 by an argument that gives the correct base in the exponential tail-bound. In many cases, one can show that this constant is arbitrarily close to the optimal constant one, that is, we get $t=k+O(T)$ stopping times. This is called perfect pipelining for the following intuitive reason: It takes a node $O(T)$ time to receive the first message and only $k$ steps later it has received all $k$ messages-as if the messages had been perfectly lined up.

We demonstrate this on the synchronous BROADCAST from Section 6.3. The proof is a bit more technical than most proofs in this article.

LEMMA 7.1. With high probability, the synchronous broadcast gossip protocol that uses logarithmic size coding coefficients, that is, $\log q=\Omega(\log n)$, takes at most $k+O(T)$ rounds to spread $k$ messages, where $T=\frac{\log (n h)}{h}$ if the isoperimetric number of the graph $G(t)$ is at least hat any time $t$.

The following proposition is needed for the proof of Lemma 7.1:

Proposition 7.2. Let $X_{1}, X_{2}, \ldots, X_{l}$ be i.i.d. Bernoulli variables with probability $P\left(X_{1}=0\right)=p \leq \frac{1}{2}$. The probability that a positively weighted sum of the variables is at most $\frac{1}{4}$ its expectation is at most $p$, that is:

$$
\forall w_{1}, \ldots, w_{l}>0: \quad P\left(\sum_{j} w_{j} X_{j} \leq \frac{1}{4}(1-p) \sum_{j} w_{j}\right) \leq p .
$$

Proof. We first scale the weights such that $\sum_{j} w_{j}=1$ and then use the second moment method:

$$
\begin{aligned}
& P\left(\sum_{j} w_{j} X_{j} \leq \frac{1}{4}(1-p)\right) \\
& \quad=P\left(\sum_{j} w_{j}\left(1-X_{j}\right)-p \sum_{j} w_{j} \geq 1-\frac{1}{4}(1-p)-p\right) \\
& \quad=P\left(\sum_{j} w_{j}\left(1-X_{j}\right)-p \sum_{j} w_{j} \geq \frac{3}{4}(1-p)\right) \\
& \quad=P\left(\left(\sum_{j} w_{j}\left(1-X_{j}\right)-p \sum_{j} w_{j}\right)^{2} \geq \frac{9}{16}(1-p)^{2}\right) .
\end{aligned}
$$


Now the left-hand side is the variance of a weighted sum of i.i.d. Bernoulli variables with probability $1-p$, and as such its expectation is exactly $\sum_{j} w_{j}^{2}(1-p) p$. Using Markov's inequality on this expectation, we get that the probability we want to bound is at most:

$$
\begin{aligned}
\left(\sum_{j} w_{j}^{2}(1-p) p\right)\left(\frac{9}{16}(1-p)^{2}\right)^{-1} & =\frac{16}{9} \frac{p}{1-p} \sum_{j} w_{j}^{2} \\
& \leq \frac{16}{9} \cdot 2 p \cdot 1 / 4 \leq p .
\end{aligned}
$$

The last transformation holds because $1-p \geq 1 / 2$ and because we can assume that all weights are at most $1 / 4$. This is true because if there is a $w_{i} \geq 1 / 4$, then already $X_{i}=1$ leads to an outcome of at least one fourth of the expectation and the probability for this to happen is $p$.

Proof of Lemma 7.1. We modify the proof of Lemma 6.4 only in the way that we use a slightly more precise tail bound, namely that the probability that after $t=k+O(T)$ independent trials there are less than $T$ successes is at most $p^{k}$, where $p$ is the failure probability (as long as $-\log p \geq \Omega(\log t)$ ). To see this, we pick $t=k-(T+1) \log t / \log p+$ $T$ and get that

$$
p^{k}=p^{t-T} t^{T+1}>\sum_{i=t-T}^{t}\left(\begin{array}{c}
t \\
t-i
\end{array}\right) p^{i}(1-p)^{t-i},
$$

which is exactly the probability for having at least $t-T$ failures in $t$ rounds.

We keep the same definition of success as in the proof of Lemma 6.4 but prove that the success probability of a round is at least $1-1 / q$, instead of $1 / 4$ as in Lemma 6.4:

If only one node is supposed to learn for a success, this is again clear by Lemma 4.2. If at least $\lceil i\rceil$ nodes are needed to a success, then we know also, by the definition of a success, that at least $4\lceil i\rceil$ nodes that do not know $\vec{\mu}$ are connected to a node that knows about it. We assign each ignorant node to exactly one node that knows about $\vec{\mu}$, breaking ties arbitrarily. Now, according to Lemma 4.2, with probability $1-1 / q$ each such node independently sends out a message that is not perpendicular to $\vec{\mu}$. In this case, all ignorant nodes connected to it learn $\vec{\mu}$. We can now directly apply Lemma 7.2 and obtain that we indeed have a success probability of at least $1-1 / q$ per round. This finishes the proof.

Another interesting case to analyze the leading constant of $k$ is the random phone call process from Section 6.1. The authors of Deb et al. [2006] gave a theoretical analysis in the regime where the $O(k)$ term dominates and proved an upper bound of 3.46k for the PUSH protocol and 5.96k for the PULL model. They also simulated the protocol and estimated the stopping time to be $1.5 k+\log _{2} n$. Both their analytic bounds and the simulation assume that messages start out in separate nodes and are equally spread over the network (see also Section 7.1).

In this section we improve over these findings and show that the PULL model in this setting actually converges in $(1+o(1)) k$ time if the number of messages $k$ is only minimally larger to dominate the running time (i.e., $k>\log ^{1+o(1)} n$ ). Interestingly, we also show that with a worst-cast initialization (see also Section 7.1) the PULL model does not achieve this convergence time but has a provably worse leading constant between 1.58 and 1.83 .

We note that determining the correct constants for the random phone call model is more delicate than proving order optimal convergence times. The reason for this is that 
the union of random exchanges over many rounds almost surely forms an expander while the graph in a single round is usually not even connected. This is the case for all of the presented random phone call models. This results in all these models being very robust order optimal while the leading constant is very sensitive to protocol details that make proving optimal $k(1+o(1))$-type bounds for large $k$ a bit more tricky. We exemplify this by exemplifying some of these concerns for the PULL protocol.

The worst-case initialization for the PULL protocol is when all messages are initially known to only one node. In this case, this node is not pulled at all in one round with probability $(1-1 / n)^{n} \approx e^{-1}=0.367879441$. In order to get pulled at least $k$ times, it takes, therefore, in expectation at least $k /\left(1-e^{-1}\right)=1.58197671 k$ rounds. Thus, for the case where only one node initially knows about all messages and if this node prepares a message in each round, which it sends out to the nodes requesting it, then this is an information-theoretic lower bound on the number of rounds. A direct analysis of the protocol for this case gives a constant of $\log (q) / \log ((1 / e+(1-1 / e) / q))$, which is $1.82462135 k$ for $q=2$. This can be improved if the start state is more mixed: The next lemma shows a $(1+o(1)) k$ stopping time for the case where all messages are initiated at different nodes. This contrasts the upper bound of 5.96k and the estimate of $1.5 k$ of Deb et al. [2006] for exactly this setting. More extensive simulation results than the ones in Deb et al. [2006] done by the author confirm that the constant for the dependency on $k$ should indeed be smaller than the projected $1.5 k$.

LEMMA 7.3. The RLNC algorithm in the random phone call PULL model even with $q=2$ spreads $k=\log ^{1+o(1)} n$ messages with high probability in $(1+o(1)) k$ time if all messages are initially known to different nodes.

Proof. We assume that each message is initially known to exactly one node and all messages are known to different nodes. This implies that exactly the $\left(\begin{array}{c}k \\ i\end{array}\right)(q-1)^{i}$ vectors that have $i$ non-zero components are initially known to exactly $i$ nodes. We will prove that any running time $t$ with $t>k+O(\log n) \log t$ suffices to spread all messages with probability at least $1-n^{-\Omega(1)}$. For the assumed case that the number of messages $k$ is with $\log ^{1+o(1)} n$ large enough for $k$ to dominate the running time, this is can then be chosen to be $t=(1+o(1)) k$.

For the proof, we pick a small threshold $f=\omega(1)$ and first look at the $\sum_{i=1}^{f}\left(\begin{array}{c}k \\ i\end{array}\right)(q-1)^{i} \leq$ $f k^{f}$ vectors that are known to at most $f$ nodes initially. From the proof of Lemma 6.1, we know that, after $t$ rounds, each of these vectors has a probability of at most $2^{-\Theta(t-O(\log n))}$ to not have spread completely. Choosing $t>k+O(\log n)$, therefore, suffices easily to make the contribution of these vectors to the union bound at most $n^{-\Omega(1)}$.

Most of the $q^{k}$ vectors start initially known to at least $f$ nodes. For these vectors $\vec{\mu}$, we choose the same definition of success as in the proof of Lemma 6.1: A round is successful if the number of nodes that know $\vec{\mu}$ increases by at least a constant factor $\lambda>1$ or if the number of nodes that do not know $\vec{\mu}$ decreases by a factor of $\lambda$. We will show that if we choose $\lambda$ small enough these vectors have a probability of $\frac{1}{q}$ to spread successfully in one round.

While with our initial analysis the start phase was the critical bottleneck, we can show that the success probability for this phase can now even be pushed below $1 / q$ by choosing $\lambda$ small enough. In the first phase, we have $k<n / 2$ nodes that know $\vec{\mu}$ and at least $n / 2$ nodes that are pulling for it. Each of those nodes has an independent probability of $k / n$ to hit a knowing node. Because $k \geq f$ we have that the probability that none of these nodes pulls from a node knowing about $\vec{\mu}$ is $(1-k / n)^{n / 2}<e^{-k} \leq e^{-f}=o(1)$. Lemma 4.2 shows that each node that does pull from a node that knows about $\vec{\mu}$ has a probability of $(1-1 / q)=1 / 2$ to learn $\vec{\mu}$. This means, more generally, that we have at 
least $n / 2$ nodes that have an independent chance of $k / 2 n$ to learn $\vec{\mu}$. For a small-enough $\lambda$ it is clear that the probability that at least $\lambda k$ nodes learn about $\vec{\mu}$ can be made an arbitrarily small constant.

In the second phase, there are at least $n / 2$ nodes that know $\vec{\mu}$ and, we want that of the remaining $k \leq n / 2$ nodes at least a $\lambda$-fraction learns $\vec{\mu}$. Each of these nodes has a probability of at least $1 / 2(1-1 / q)$ to pull from a knowing node and learn $\vec{\mu}$ (see Lemma 4.2). Choosing $\lambda=1 / 8$ suffices to guarantee that the probability that at least a $\lambda$-fraction learns $\vec{\mu}$ is at least $1 / 2$. The only reason that this probability cannot be reduced is because if only one node remains to learn about $\vec{\mu}$, then a round is successful with probability exactly $1 / 2$.

Now let $T=O(\log n)$ be the number of successful rounds needed to spread $\vec{\mu}$. Similarly to the proof Lemma 7.1 , we approximate the probability that $\vec{\mu}$ has not spread after $t$ round by:

$$
\sum_{i=t-T}^{t}\left(\begin{array}{c}
t \\
t-i
\end{array}\right) \frac{1}{2^{i}}\left(1-\frac{1}{2}\right)^{t-i} \leq t^{T} \frac{1^{t}}{2} .
$$

Thus, if $t>k+T \log t$ as desired this probability is smaller than $2^{-k} n^{-\Omega(1)}$ and a union bound over all vectors finished the proof by showing that the probability that after $t$ rounds not all vectors have spread is at most $n^{-\Omega(1)}$.

\subsection{Asynchronous Single Transfer with Small $k$}

Section 6.2 proves convergence times for spreading $k=n$ messages using the asynchronous single transfer protocols. These bounds are tight and directly extend to a $\Theta\left(\frac{k}{\gamma}\right)$ bound for $k=\Omega(n)$ messages. In what follows, we want to generalize this to smaller number of messages and discuss the bounds that can be obtained using the projection analysis technique.

For small number of messages, for example, $k=1$, the convergence time of RLNC single transfer gossip can be much faster than $O\left(\frac{n}{\gamma}\right)$ but still be $\omega\left(\frac{k}{\gamma}\right)$. This shows that the min-cut $\gamma$ is not the right quantity to look at in this scenario. Again, as in Section 6.3, conductance quantities capture much better how fast a small number of messages spreads. The quantity we consider is:

$$
\lambda(G)=\min _{\emptyset \neq S \subset V} \frac{\sum_{e \in \operatorname{out}(S)} p_{e}}{\min (|S|,|\bar{S}|)} .
$$

It is easy to see that it takes at most $T=O\left(\frac{\log n}{\lambda}\right)$ time for one message to spread if the conductance is bounded by $\lambda$ at every time $t$.

THEOREM 7.4. In the asynchronous single transfer model (with any q) it takes in expectation at most $T=O\left(\frac{\log n}{\lambda}\right)$ time for one message to spread.

Proof. The probability that a set of nodes that know the message grows from size $i<n$ to $i+1$ is at least $(1-1 / q)(\lambda \min (k, n-k))$. It thus takes at least $\frac{1}{(1-1 / q) \lambda}$ rounds in expectation for the first success, $\frac{1}{2(1-1 / q) \lambda}$ rounds for the second success, and, in general, $T=\sum_{i=1}^{n} \frac{1}{\min (i, n-i)(1-1 / q) \lambda}<\left(1+\frac{1}{q-1}\right) \frac{2}{\lambda} \log n=O\left(\frac{\log n}{\lambda}\right)$ rounds in expectation for one message to spread.

This is a tight bound for many regular graphs and gives, for example, a convergence time of $\Theta(n \log n)$ for the complete graph or any other regular expanders. The other lower bound that kicks in for large enough $k$ is the $\Omega\left(\frac{k}{\gamma}\right)$ lower bound from Lemma 6.3. 
Similarly to the results for the other models, the next lemma nicely demonstrates that the convergence time (up to a small additional $\log n$-factor that falls out of our elementary analysis) is again either dominated by the number of rounds needed to spread one message or, for larger number of messages $k$, by the $\frac{k}{\gamma}$ rounds needed for the $k$ messages to cross the worst case cut.

LEMMA 7.5. With high probability, disseminating $k$ messages in the asynchronous single transfer model with $q=2$ takes at most $t=O\left(\frac{k}{\gamma}+\frac{\log ^{2} n}{\lambda}\right)$ rounds if the graph $G$ has a min-cut of at most $\gamma$ and a conductance of at least $\lambda$ at all times $t$.

PRoof. We want to show that running the protocol for $t=O\left(\frac{k}{\gamma}+T\right)$ rounds, where $T=O\left(\frac{\log ^{2} n}{\lambda}\right)$ suffices to spread $k$ messages. Note that we always have $t>n$ and can also safely assume that $\log t=O(\log n)$. As a first step, we define $p_{i}$ to be a lower bound for the probability that if $i$ nodes know $\vec{\mu}$ in the next round, then one more node learns about $\vec{\mu}$. Note that by assumption and Lemma $4.2 p_{i}$ is lower bounded by $(1-1 / q) \min \{i, n-i\} \lambda$ and $(1-1 / q) \gamma$. We now look at $n$ phases in which we allow $\frac{\ln 3 t}{p_{i}}$ tries for $i$ nodes informing the next node about $\vec{\mu}$. The number of rounds spent in successful phases sums up to at most $\sum_{i=1}^{n} \frac{\ln 3 t}{p_{i}} \leq O\left(\frac{\log n}{\lambda}\right) \sum_{i=1}^{n / 2} \frac{1}{i} \leq O\left(\frac{\log ^{2} n}{\lambda}\right)=T$. Let us now look at the probability that $\vec{\mu}$ has not spread after $t>T$ steps. In this case we have at least $t-T$ failures that can occur after any of the $n$ phases. The probability that at least $m$ errors occur after phase $i$ is at most $\left(1-p_{i}\right)^{\frac{\ln 3 t}{p_{i}}+m}<(3 t)^{-1}(1-\gamma / 2)^{m}$. We thus get a $(2 t)^{-1}$ factor for every phase that does not finish "in time." We also get a total factor of $(1-\gamma / 2)^{t-T}$ from all $t-T$ failures occurring after any round. Let $j$ be the number of phases that finish not "in time." There are exactly $\left({ }_{j}^{(t-T)+j}\right)<(2 t)^{j}$ ways of distributing the $t-T$ failures to these $j$ phases. Putting all this together, we get that the probability that the algorithm did not converge after $t>T$ steps is upper bounded by:

$$
\sum_{j=1}^{n}(2 t)^{j}(3 t)^{-j}(1-\gamma / 2)^{t-T} \leq e^{-\gamma / 2(t-T)} .
$$

Choosing $t=O\left(\frac{k}{\gamma}+T\right)$ makes this smaller than $q^{-k} 2^{-n}$ and applying Theorem 4.4 completes the proof.

\subsection{Weaker Requirements for Random Networks}

The idea behind proving performances in the extremely strong and pessimistic adaptive adversarial model used in this article is that the guarantees directly extend to the widest possible range of dynamic networks, including random models. Most of our proofs, like the ones of Lemmas 6.2,6.4, or 6.5, demand that the network graph $G(t)$ has a certain connectivity requirement at any time $t$. These requirements might be slightly too strong for random network models. In the following, we briefly discuss how these requirements can be easily weakened in many ways:

The simple fact that no progress in the spreading of knowledge gets lost makes it easy to deal with the case that the connectivity fluctuates (e.g., randomly). Increasing the stopping time by a constant factor easily accounts for models in which the desired connectivity occurs only occasionally or with constant probability. Looking at the average connectivity is another possibility. It is furthermore not necessary to require the entire graph to be expanding on average but it suffices to demand that each subset expands on average with constant probability according to its size. This way, convergence can be proven even for always-disconnected graphs (see Haeupler and Médard 
[2011] for several examples and proofs). In many models, it is furthermore possible and helpful to consider the union of the network graphs of consecutive rounds, that is, $G^{\prime}(t)=G\left(3 t^{\prime}\right) \cup G\left(3 t^{\prime}+1\right) \cup G\left(3 t^{\prime}+2\right)$.

We demonstrate the usefulness of these ideas on a small example, namely, an alternative way to prove Lemma 6.1: Instead of analyzing the random phone call model as a gossip process on the complete graph, one can alternatively see it as a synchronous BROADCAST on a random network. The network graph $G(t)$ is in this case simply formed by a random directed in-edge, directed out-edge, or undirected edge at each node, depending on whether the PUSH, PULL, or EXCHANGE model is used. For these random topologies $G(t)$, Lemma 6.4 will not directly give any stopping time bound, simply because the network graph $G(t)$ is with high probability disconnected. Using either of the two ideas introduced above solves this problem: With constant probability, every set has a constant expansion; alternatively, one can use that the union of a constant number of rounds forms most likely with an expander.

\section{CONCLUSIONS AND OPEN QUESTIONS}

We introduced the projection analysis technique as a new way to analyze (gossip) protocols based on random linear network coding. Our analysis drastically simplifies, extends, and strengthens previous results. In all settings considered in this article, we prove that the RLNC gossip spreads messages in a perfectly pipelined manner and stops with high probability in optimal time. As a most notable extension, all our results hold in highly dynamic networks that are controlled by a fully adaptive adversary.

Theorem 4.4 gives a direct way to transfer results for a single-message gossip process to the corresponding multi-message RLNC gossip process, given that strong-enough tail bounds are provided. For example, applying Theorem 4.4. to the results of [Chierichetti et al. 2010; Giakkoupis 2011, 2014] leads to tight running time bounds for RLNC gossip on edge- and vertex-expanders. Another interesting candidate for transferring results from gossip with one message to RLNC gossip could be the edge-Markovian evolving graphs studied in Clementi et al. [2010].

The projection analysis technique is quite simple and flexible, and we expect that it has more applications and extensions. Since the initial submission of this article, the author, in collaboration with Médard, Karger, Cohen, Avin, and Kim, demonstrated some of these: Among other things, Haeupler and Karger [2011] show that RLNC, with a large-enough $q$, continues to work optimally against omniscient adversaries. This also leads to a (non-uniform) derandomization. Haeupler and Médard [2011] use the projection technique to show that optimal stopping times are preserved if nodes keep only one instead of all packets for further coding purposes. Haeupler et al. [2011] shows, using techniques based on Ho et al. [2006], that in any model with an oblivious adversary RLNC stops in exactly optimal time, that is, when there is enough connectivity in the time-expanded graph. This implies that the projection technique can be seen as proving tight bounds on the connectivity of many network models, an important but usually very challenging task. Haeupler et al. [2012] further extends the projection analysis and shows that it can also be used to analyze gossip with correlated inputs. Last, Alon et al. [2014], Ghaffari and Haeupler [2013], Censor-Hillel et al. [2012b], and Censor-Hillel et al. [2015] demonstrate the power of the projection analysis technique in analyzing network coding protocols in (distributed) radio networks.

\section{REFERENCES}

D. Agrawal, A. El Abbadi, and R. C. Steinke. 1997. Epidemic algorithms in replicated databases (extended abstract). In Proceedings of the Symposium on Principles of Database Systems (PODS). 161-172.

R. Ahlswede, N. Cai, S. Y. R. Li, and R. W. Yeung. 2000. Network information flow. Trans. Informat. Theor. 46, 4 (2000), 1204-1216. 
Noga Alon, Mohsen Ghaffari, Bernhard Haeupler, and Majid Khabbazian. 2014. Broadcast throughput in radio networks: Routing vs. network coding. In Proceeding of the ACM Symposium on Discrete Algorithms (SODA). ACM, New York, NY, 1831-1843. DOI : http://dx.doi.org/10.1137/1.9781611973402.132

James Aspnes and Eric Ruppert. 2007. An introduction to population protocols. Bulletin of the European Association for Theoretical Computer Science, Distributed Computing Column, 93 (Oct. 2007), 98-117.

Chen Avin, Michal Kouckỳ, and Zvi Lotker. 2008. How to explore a fast-changing world (cover time of a simple random walk on evolving graphs). In Proceedings of the International Conference on Automata, Languages and Programming (ICALP). 121-132.

M. Borokhovich, C. Avin, and Z. Lotker. 2010. Tight bounds for algebraic gossip on graphs. In Proceedings of the IEEE International Symposium on Information Theory (ISIT). IEEE, Los Alamitos, CA, 1758-1762.

Keren Censor-Hillel, Bernhard Haeupler, Jonathan Kelner, and Petar Maymounkov. 2012a. Global computation in a poorly connected world: Fast rumor spreading no dependence on conductance. In Proceeding of the ACM Symposium on Theory of Computing (STOC). ACM, Nw York, NY, 961-970. DOI : http://dx.doi.org/10.1145/2213977.2214064

Keren Censor-Hillel, Bernhard Haeupler, Nancy Lynch, and Muriel Médard. 2012b. Bounded-contention coding for wireless networks in the high SNR regime. In Proceeding of the Symposium on Distributed Computing (DISC). 91-105. DOI : http://dx.doi.org/10.1007/978-3-642-33651-5_7

Keren Censor-Hillel, Bernhard Haeupler, Nancy Lynch, and Muriel Médard. 2015. Boundedcontention coding for the additive network model. Distributed Computing (DIST) (2015), 1-12. DOI : http://dx.doi.org/10.1007/s00446-015-0244-9

F. Chierichetti, S. Lattanzi, and A. Panconesi. 2010. Almost tight bounds for rumour spreading with conductance. In Proceedings of the ACM Symposium on Theory of Computing (STOC). ACM, New York, NY, 399-408.

P. A. Chou, Y. Wu, and K. Jain. 2003. Practical network coding. In Proceedings of the Allerton Conference on Communication Control and Computing. 40-49.

Andrea Clementi, Claudio Macci, Angelo Monti, Francesco Pasquale, and Riccardo Silvestri. 2010. Flooding time of edge-markovian evolving graphs. SIAM J. Discr. Math. 24, 4 (2010), 1694-1712.

Supratim Deb and Muriel Médard. 2004. Algebraic gossip: A network coding approach to optimal multiple rumor mongering. In Proceedings of the Allerton Conference on Communication, Control, and Computing.

S. Deb, M. Médard, and C. Choute. 2005. On random network coding based information dissemination. In Proceedings of the IEEE International Symposium on Information Theory (ISIT). IEEE, Los Alamitos, CA, 278-282.

S. Deb, M. Médard, and C. Choute. 2006. Algebraic gossip: A network coding approach to optimal multiple rumor mongering. Trans. Informat. Theor. (TransInf) 52, 6 (2006), 2486-2507.

A. Demers, D. Greene, C. Hauser, W. Irish, J. Larson, S. Shenker, H. Sturgis, D. Swinehart, and D. Terry. 1987. Epidemic algorithms for replicated database maintenance. In Proceedings of the ACM Symposium on Principles of Distributed Computing (PODC). ACM, New York, NY, 1-12.

Chinmoy Dutta, Gopal Pandurangan, Rajmohan Rajaraman, Zhifeng Sun, and Emanuele Viola. 2013. On the complexity of information spreading in dynamic networks. In Proceedings of the ACM Symposium on Discrete Algorithms (SODA). ACM, New York, NY, 717-736.

Uriel Feige, David Peleg, Prabhakar Raghavan, and Eli Upfal. 1990. Randomized broadcast in networks. Rand. Struct. Algor. 1, 4 (1990), 447-460.

C. Fragouli, J. Le Boudec, and J. Widmer. 2006. Network coding: An instant primer. Comput. Commun. Rev. 36,1 (2006), 63.

Christina Fragouli, Jörg Widmer, and Jean-Yves Le Boudec. 2008. Efficient broadcasting using network coding. Trans. Network. 16, 2 (2008), 450-463.

Mohsen Ghaffari and Bernhard Haeupler. 2013. Fast structuring of radio networks for multi-message communications. In Proceeding of the Symposium on Distributed Computing (DISC). 492-506. DOI : http://dx.doi.org/10.1007/978-3-642-41527-2_34

George Giakkoupis. 2011. Tight bounds for rumor spreading in graphs of a given conductance. In Proceedings of the Symposium on Theoretical Aspects of Computer Science (STACS). 57-68.

George Giakkoupis. 2014. Tight bounds for rumor spreading with vertex expansion. In Proceedings of the ACM Symposium on Discrete Algorithms (SODA). ACM, New York, NY, 801-815.

George Giakkoupis, Thomas Sauerwald, and Alexandre Stauffer. 2014. Randomized rumor spreading in dynamic graphs. Proceedings of the International Conference on Automata, Languages, and Programming (ICALP) (2014), 495-507.

C. Gkantsidis and P. R. Rodriguez. 2005. Network coding for large scale content distribution. In Proceedings of the International Conference on Computer Communications (INFOCOM), vol. 4. 2235-2245. 
Zygmunt J. Haas, Joseph Y. Halpern, and Li Li. 2006. Gossip-based ad hoc routing. Trans. Network. 14, 3 (2006), 479-491. DOI : http://dx.doi.org/10.1109/TNET.2006.876186

Bernhard Haeupler. 2011. Analyzing network coding gossip made easy. In Proceeding of the ACM Symposium on Theory of Computing (STOC). ACM, New York, NY, 293-302. DOI:http://dx.doi.org/ 10.1145/1993636.1993676

Bernhard Haeupler. 2012. Tighter worst-case bounds on algebraic gossip. IEEE Commun. Lett. 16, 8 (2012), 1274-1276.

Bernhard Haeupler. 2013. Simple, fast, and deterministic gossip and rumor spreading. In Proceeding of the ACM-SIAM Symposium on Discrete Algorithms (SODA). 705-716.

Bernhard Haeupler, Asaf Cohen, Chen Avin, and Muriel Médard. 2012. Network coded gossip with correlated data. In Proceeding of the IEEE International Symposium on Information Theory (ISIT). IEEE, Los Alamitos, CA, 2616-2620. DOI :http://dx.doi.org/10.1109/ISIT.2012.6283992

Bernhard Haeupler and David Karger. 2011. Faster information dissemination in dynamic networks via network coding. In Proceedings of the ACM Symposium on Principles of Distributed Computing (PODC). ACM, Los Alamitos, CA, 381-390.

Bernhard Haeupler, MinJi Kim, and Muriel Médard. 2011. Optimality of network coding with buffers. In Proceedings of the IEEE Information Theory Workshop (ITW). 533-537.

Bernhard Haeupler and Fabian Kuhn. 2012. Lower bounds on information dissemination in dynamic networks. In Proceeding of the International Symposium on Distributed Computing (DISC). 166-180. DOI : http://dx.doi.org/10.1007/978-3-642-33651-5_12

Bernhard Haeupler and Dahlia Malkhi. 2014. Optimal gossip with direct addressing. In Proceeding of the ACM Symposium on Principles of Distributed Computing (PODC). 176-185. DOI:http://dx.doi.org/ 10.1145/2611462.2611489

Bernhard Haeupler and Muriel Médard. 2011. One packet suffices - highly efficient packetized network coding with finite memory. In Proceedings of the IEEE International Symposium on Information Theory (ISIT). IEEE, Los Alamitos, CA, 1151-1155.

S. M. Hedetniemi, S. T. Hedetniemi, and A. L. Liestman. 1988. A survey of gossiping and broadcasting in communication networks. Networks 18 (1988), 319-349.

T. Ho, M. Médard, R. Koetter, D. R. Karger, M. Effros, J. Shi, and B. Leong. 2006. A random linear network coding approach to multicast. Trans. Informat. Theor. 52, 10 (2006), 4413-4430.

R. Karp, C. Schindelhauer, S. Shenker, and B. Vöcking. 2000. Randomized rumor spreading. In Proceedings of the Symposium on Foundations of Computer Science (FOCS). 565-574.

S. Katti, H. Rahul, W. Hu, D. Katabi, M. Médard, and J. Crowcroft. 2008. XORs in the air: Practical wireless network coding. Trans. Network. 16, 3 (2008), 497-510.

D. Kempe, A. Dobra, and J. Gehrke. 2003. Gossip-based computation of aggregate information. In Proceedings of the IEEE Symposium on Foundations of Computer Science (FOCS). IEEE, Los Alamitos, CA, $482-491$.

D. Kempe, J. Kleinberg, and A. Demers. 2004. Spatial gossip and resource location protocols. J. ACM 51, 6 (2004), 943-967.

Fabian Kuhn, Nancy Lynch, and Rotem Oshman. 2010. Distributed computation in dynamic networks. In Proceedings of the ACM Symposium on Theory of Computing (STOC). ACM, New York, NY, 557-570.

S. Y. R. Li, R. W. Yeung, and N. Cai. 2003. Linear network coding. Trans. Informat. Theor. 49, 2 (2003), $371-381$.

Y. Minski. 2002. Spreading Rumors Cheaply, Quickly, and Reliably. Ph.D. Dissertation. Cornell University.

D. Mosk-Aoyama and D. Shah. 2006a. Computing separable functions via gossip. In Proceedings of the ACM Symposium on Principles of Distributed Computing. ACM, New York, NY, 113-122.

D. Mosk-Aoyama and D. Shah. 2006b. Information dissemination via network coding. In Proceedings of the IEEE International Symposium on Information Theory. IEEE, Los Alamitos, 1748-1752.

D. Vasudevan and S. Kudekar. 2009. Algebraic gossip on Arbitrary Networks. ArXiv:0901.1444 (2009).

Received September 2011; revised August 2015; accepted January 2016 